\title{
Effect of film thickness on the thermal resistance of confined semiconductor thin films
}

\author{
E. S. Landry and A. J. H. McGaughey ${ }^{a)}$ \\ Department of Mechanical Engineering, Carnegie Mellon University, Pittsburgh, Pennsylvania 15213, USA
}

(Received 12 October 2009; accepted 25 November 2009; published online 15 January 2010)

\begin{abstract}
The thermal resistance of semiconductor thin films is predicted using lattice dynamics (LD) calculations and molecular dynamics (MD) simulations. We consider Si and Ge films with thicknesses, $L_{F}$, between 0.2 and $30 \mathrm{~nm}$ that are confined between larger extents of the other species (i.e., Ge/Si/Ge and $\mathrm{Si} / \mathrm{Ge} / \mathrm{Si}$ structures). The LD predictions are made in the classical limit for comparison to the classical MD simulations, which are performed at a temperature of $500 \mathrm{~K}$. For structures with $L_{F}<2 \mathrm{~nm}$, the thin film thermal resistance increases rapidly with increasing film thickness, a trend we attribute to changes in the allowed vibrational states in the film. These changes are found to affect the dependence of the phonon transmission coefficient on incidence angle for the $\mathrm{Ge} / \mathrm{Si} / \mathrm{Ge}$ structures and on frequency for the $\mathrm{Si} / \mathrm{Ge} / \mathrm{Si}$ structures. When $L_{F}>2 \mathrm{~nm}$, the MD-predicted thermal resistances are independent of the film thickness for the $\mathrm{Ge} / \mathrm{Si} / \mathrm{Ge}$ structures and increase with increasing film thickness for the $\mathrm{Si} / \mathrm{Ge} / \mathrm{Si}$ structures. We attribute these results to phonon transport that is ballistic in the $\mathrm{Ge} / \mathrm{Si} / \mathrm{Ge}$ structures and more diffusive in the $\mathrm{Si} / \mathrm{Ge} / \mathrm{Si}$ structures based on comparisons to the LD predictions, which assume ballistic phonon transport. We find that this difference between the structures cannot be predicted by comparing the mode-averaged phonon mean free path to the film thickness. It can be predicted, however, by considering the frequency dependence of the phonon mean free paths. (c) 2010 American Institute of Physics.

[doi:10.1063/1.3275506]
\end{abstract}

\section{INTRODUCTION}

Semiconductor thin films are pervasive in advanced technological devices. For example, the active region in a quantum cascade laser or a light-emitting diode contains films of direct bandgap semiconductors such as GaAs, AlGaAs, and GaN with thicknesses of $1-10 \mathrm{~nm} .{ }^{1,2}$ The $\mathrm{SiO}_{2}$ dielectric layer between the poly-Si gate and $\mathrm{Si}$ substrate in a field effect transistor can be as thin as $1 \mathrm{~nm} .^{3}$ Superlattices, periodic materials that contain films of alternating species (e.g., $\mathrm{Si}$ and $\mathrm{Ge}$ ) with thicknesses as small as $1 \mathrm{~nm}$, are being studied for their potential to increase the efficiency of thermoelectric energy conversion devices. ${ }^{4-6}$ These film thicknesses are less than the mean free paths of many of the phonons (i.e., quantized lattice vibrations that dominate the thermal transport in semiconductors) in the corresponding bulk material. The thermal transport properties of semiconductor thin films are thus different than their corresponding bulk values. ${ }^{7,8}$ To aid in the design of devices employing thin films, accurate models for the film thermal transport properties are required.

One common approach for modeling thermal transport in a semiconductor thin film is to solve the Boltzmann transport equation (BTE) ${ }^{9-12}$ In this approach, phonons are treated as particles and their wavelike nature is neglected. Closing the BTE requires specification of the phonon scattering rates (i.e., the phonon relaxation times or mean free paths) and boundary conditions. Typically, all of the phonons in the film are assumed to have a mode-independent mean

${ }^{a)}$ Electronic mail: mcgaughey@cmu.edu. free path. ${ }^{9,10}$ This assumption is difficult to justify because the phonon relaxation times in semiconductors such as $\mathrm{Si}$ are predicted to span many orders of magnitude. ${ }^{13-15}$ The boundary conditions are often specified by assuming that a fraction of the phonons scatter specularly at the boundaries between the film and its adjoining medium, which may be a solid, fluid, or vacuum, while the remainder scatter diffusely. ${ }^{10,13}$ This fraction is often treated as a mode-independent fitting parameter, thereby neglecting the atomic-level detail of the boundaries. Even with more accurate methods to close the BTE (e.g., mode-dependent phonon properties obtained from $a b$ initio calculations), the BTE-based approach will not be suitable for films with thicknesses on the same scale as the average wavelength of the thermally excited phonons, where the wavelike nature of the phonons is important. ${ }^{16}$ For $\mathrm{Si}$ at room temperature, this wavelength is estimated to be $1 \mathrm{~nm} .{ }^{16}$

Molecular dynamics (MD) simulations and lattice dynamics (LD) calculations can be used to study thermal transport in films in which the wavelike nature of the phonons is important. ${ }^{11,17-23}$ In a MD simulation, the positions and momenta of a set of atoms evolve classically according to Newton's equations of motion. Such simulations are an ideal method for predicting thermal transport properties because no assumptions concerning the phonon scattering within the film or at the film boundaries are required. Previous MDbased studies used simulation cells that contained a film confined between two thermal reservoirs of the same species. ${ }^{19-23}$ In these studies, the film thermal conductivity is predicted to increase toward the bulk value as the film thickness increases due to the decreasing effect of phonon scattering at the film/reservoir boundaries on the phonon mean 


\begin{tabular}{ll|l|lll} 
Left Lead & \multicolumn{2}{c}{$\begin{array}{c}\text { Thin Film } \\
\text { Region }\end{array}$} & \multicolumn{2}{c}{ Right Lead } \\
\hline Si & $T_{L}$ & Ge & $T_{R}$ & $\mathrm{Si}$ \\
& & & & \\
\hline
\end{tabular}
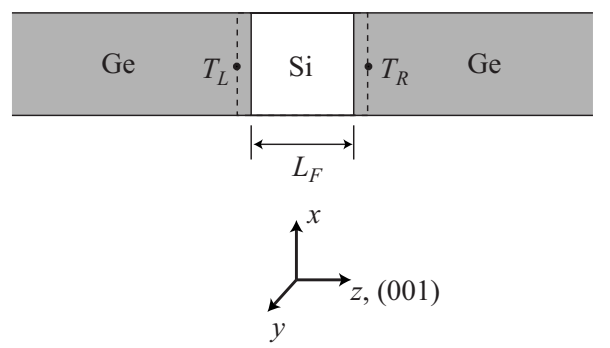

FIG. 1. Schematic diagrams of the $\mathrm{Si} / \mathrm{Ge} / \mathrm{Si}$ and $\mathrm{Ge} / \mathrm{Si} / \mathrm{Ge}$ structures.

free paths. Beyond obtaining predictions of the thermal conductivity, it is challenging to extract additional details related to the phonon transport from a MD simulation due to computational expense. ${ }^{24-26}$ These details can be more easily obtained using LD calculations, ${ }^{27}$ in which the system dynamics are transformed from the real-space coordinates (the atomic positions) to the reciprocal-space coordinates (the phonon modes). By accessing the mode-dependent phonon properties (e.g., phonon frequencies, group velocities, and transmission coefficients), a more thorough understanding of thermal transport can be obtained. Because LD calculations are typically applied under the harmonic approximation, however, no information about inelastic phonon scattering (e.g., multiphonon scattering events) can be obtained because the phonon modes are decoupled. ${ }^{17,28}$

In this work, LD calculations and MD simulations are used to examine the dependence of thin film thermal resistance on film thickness. By comparing the predictions of the two methods, we examine how the thin film thermal resistance is affected by changes in the allowed vibrational states in the film and the presence of inelastic phonon-phonon scattering in the film. We consider two types of thin film structure (see Fig. 1). In one structure, a Ge film with thickness $L_{F}$ is confined between two $\mathrm{Si}$ leads (the $\mathrm{Si} / \mathrm{Ge} / \mathrm{Si}$ structure). In the other, a Si film is confined between two Ge leads (the $\mathrm{Ge} / \mathrm{Si} / \mathrm{Ge}$ structure). Similar configurations of a thin film confined by larger extents of a different species are often encountered in application, but have received minimal theoretical attention. In the $\mathrm{Si} / \mathrm{Ge} / \mathrm{Si}$ and $\mathrm{Ge} / \mathrm{Si} / \mathrm{Ge}$ structures, the $\mathrm{Si} / \mathrm{Ge}$ interfaces are oriented along the (001) crystallographic plane and the film is symmetrically strained by setting the lattice constant in the $x$ and $y$ directions to the average of the bulk $\mathrm{Si}$ and bulk Ge lattice constants. ${ }^{29}$ The layer spacings in the $z$ direction are chosen to give zero stress in that direction. These layer spacings are found using a steepest decent approach in the LD calculations and elasticity theory in the MD simulations, as described in Ref. 18. The atomic interactions are modeled using the Stillinger-Weber interatomic potential. $^{30-32}$

\section{METHODOLOGY}

\section{A. LD calculations}

We predict the thermal resistance of the thin film region, which includes the film and the two lead/film interfaces (see Fig. 1). We refer to the thermal resistance of this region as the "thin film thermal resistance." It is defined as

$$
R=\frac{T_{L}-T_{R}}{q},
$$

where $q$ is the heat flux across the thin film region, and $T_{L}$ and $T_{R}$ are the lead temperatures at the lead/film boundaries. At the phonon-mode level, the steady-state heat flux across the thin film region is

$$
\begin{aligned}
q= & \frac{1}{(2 \pi)^{3}} \int_{L} \sum_{\nu}^{+} \hbar \omega(\boldsymbol{\kappa}, \nu) v_{z}(\boldsymbol{\kappa}, \nu) \alpha_{L \rightarrow R}(\boldsymbol{\kappa}, \nu) f_{L}(\boldsymbol{\kappa}, \nu) d \boldsymbol{\kappa} \\
& +\frac{1}{(2 \pi)^{3}} \int_{R} \sum_{\nu}^{-} \hbar \omega(\boldsymbol{\kappa}, \nu) v_{z}(\boldsymbol{\kappa}, \nu) \alpha_{R \rightarrow L}(\boldsymbol{\kappa}, \nu) f_{R}(\boldsymbol{\kappa}, \nu) d \boldsymbol{\kappa},
\end{aligned}
$$

where $L$ and $R$ denote the left and right leads, $\hbar$ is the Planck constant divided by $2 \pi, \nu$ denotes the phonon polarization, and $\boldsymbol{\kappa}, \omega$, and $v_{z}$ are the phonon wavevector, frequency, and $z$ component of the group velocity. The first (second) integral is over the first Brillouin zone of the left (right) lead, and the first (second) summation is over phonons moving in the positive (negative) $z$ direction. The mode-dependent phonon transmission coefficient, $\alpha_{L \rightarrow R}$, is defined as the fraction of the incident phonon energy that is transmitted from the left lead to the right lead (similar for $\alpha_{R \rightarrow L}$ ). The variables $f_{L}$ and $f_{R}$ are the mode-dependent phonon distribution functions in the left and right leads at the lead/film boundaries. These distribution functions are often approximated as the equilibrium Bose-Einstein distribution function, $f_{\mathrm{BE}}$, evaluated at either $T_{L}$ or $T_{R}{ }^{10,17,33-38}$ The equilibrium distribution function is

$$
f_{\mathrm{BE}}(\omega, T)=\left[\exp \left(\frac{\hbar \omega}{k_{\mathrm{B}} T}\right)-1\right]^{-1}
$$

where $k_{\mathrm{B}}$ is the Boltzmann constant. Under this approximation, $\mathrm{LD}$ and MD predictions of the thermal resistance of an isolated $\mathrm{Si} / \mathrm{Ge}$ interface (i.e., the $\mathrm{Si} / \mathrm{Ge}$ thermal boundary resistance) are in agreement to within $5 \% .^{18}$ It is well known, however, that as the average phonon transmission coefficient approaches unity, the accuracy of this approximation decreases and the LD-predicted thermal resistances become too large. ${ }^{18,39}$ We therefore expect that approximating the phonon distributions using the equilibrium distribution will be suitable for modeling a thin film structure if the average phonon transmission coefficient across the film is less than or equal to that across the isolated $\mathrm{Si} / \mathrm{Ge}$ interface. This condition is met when the thin film thermal resistance exceeds the $\mathrm{Si} / \mathrm{Ge}$ thermal boundary resistance. Under this approximation, Eq. (2) can be substituted into Eq. (1), yielding an expression for the thin film thermal resistance that can be simplified to (see Ref. 18 for details) 


$$
R_{\mathrm{LD}}=\left[\frac{1}{(2 \pi)^{3}} \int_{L} \sum_{\nu}^{+} \hbar \omega v_{z} \alpha_{L \rightarrow R} \frac{d f_{\mathrm{BE}}}{d T} d \boldsymbol{\kappa}\right]^{-1} .
$$

We evaluate $R_{\mathrm{LD}}$ for the thin film structures using Monte Carlo integration with $10^{5}$ random phonon wavevectors in the first Brillouin zone of the left lead, where the lead is defined using the two-atom diamond primitive cell. This level of Brillouin zone sampling results in a prediction uncertainty of $\pm 2 \%$. For comparison to the classical MD predictions, Eq. (4) is evaluated in the classical limit by setting $f_{\mathrm{BE}}=k_{\mathrm{B}} T / \hbar \omega$. At each wavevector, the frequencies and group velocities are obtained using LD calculations applied under the harmonic approximation. The phonon transmission coefficients are obtained using the harmonic LD-based scattering boundary method. ${ }^{36,40,41}$ This method assumes that (i) no inelastic phonon scattering occurs within the thin film region, (ii) the phonons scatter specularly at the lead/film boundaries, and (iii) the phonons are spatially delocalized. The assumption of no inelastic scattering is valid when the phonon scattering at the $\mathrm{Si} / \mathrm{Ge}$ interfaces is elastic and the phonons travel ballistically across the film. In our previous work, we found that the $\mathrm{Si} / \mathrm{Ge}$ thermal boundary resistance is temperature independent at temperatures less than or equal to $500 \mathrm{~K}$, indicating that the first condition is met at these temperatures. ${ }^{18}$ The second condition is met when the film thickness is less than the phonon mean free paths in the corresponding bulk material. This point is discussed further in Sec. III C 2. The assumption of specular scattering is valid for our thin film structures because they contain no defects or roughness that would promote diffuse scattering. ${ }^{13,42}$ As discussed by Schelling and Phillpot, ${ }^{43}$ in order to properly model the interference between phonons reflected from the lead/film interfaces when the phonon transport is ballistic, the extent of the phonon wave packet should be greater than the film thickness. The assumption that the phonons are spatially delocalized is thus appropriate. Full details related to our LD calculations can be found elsewhere. ${ }^{18,27,44,45}$

\section{B. MD simulations}

To examine the effects of phonon-phonon scattering in the film, we compare the LD-predicted thermal resistances to values predicted using MD simulations, which require no assumptions about the nature of the phonon scattering. The MD predictions are made at a temperature of $500 \mathrm{~K}$, where the phonon scattering at the $\mathrm{Si} / \mathrm{Ge}$ interfaces is elastic. ${ }^{18}$ The velocity Verlet algorithm with a time step of $0.55 \mathrm{fs}$ is used to numerically integrate the Newtonian equations of motion. The thin film thermal resistance is predicted using the direct method, in which a known heat flux is applied across the sample, the resulting steady-state temperature drop across the film is specified, and the thermal resistance is determined from Eq. (1).

A schematic diagram of our direct method simulation cell is shown in Fig. 2(a). The system consists of either a $\mathrm{Si} / \mathrm{Ge} / \mathrm{Si}$ or $\mathrm{Ge} / \mathrm{Si} / \mathrm{Ge}$ structure that is bordered by hot and cold reservoirs of length $L_{\text {res }}=50$ monolayers and fixed boundaries in the $z$ direction. The fixed boundary regions each contain four monolayers of fixed atoms, and the left and
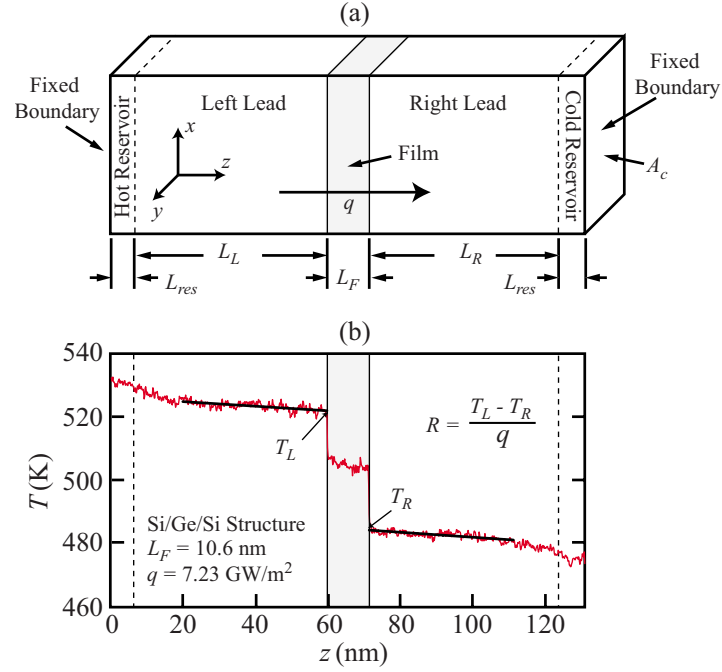

FIG. 2. (Color online) (a) Direct method simulation cell. (b) Temperature profile for the $\mathrm{Si} / \mathrm{Ge} / \mathrm{Si}$ structure with $L_{F}=10.6 \mathrm{~nm}$.

right leads have lengths $L_{L}=L_{R}=400$ monolayers. The cross section of the simulation cell is square and has area $A_{c}$ equal to four unit cells by four unit cells. Based on the results of our previous examination into the effect of simulation cell size on the $\mathrm{Si} / \mathrm{Ge}$ thermal boundary resistance, ${ }^{18}$ we expect that these dimensions will generate size-independent predictions for the thin film thermal resistance. Periodic boundary conditions are imposed in the $x$ and $y$ directions.

The sample and reservoirs are initially set to a uniform temperature by scaling the atomic velocities for $0.55 \mathrm{~ns}(1$ $\times 10^{6}$ time steps). A heat flux of $q=7.23 \mathrm{GW} / \mathrm{m}^{2}$ is then applied by adding a constant amount of kinetic energy to the hot reservoir and removing the same amount of kinetic energy from the cold reservoir at every time step using the method described by Ikeshoji and Hafskjold. ${ }^{46}$ This value of the heat flux does not influence the predicted thermal resistance.$^{18}$ From this point, a period of $3.3 \mathrm{~ns}$ is allowed to reach steady-state conditions. By examining the evolution of the temperature profile during this period, we confirmed that steady-state conditions are met for each thin film structure. After steady-state conditions are reached, the steady-state temperature profile is obtained by averaging the temperature of each monolayer over an additional $2.75 \mathrm{~ns}$. An example temperature profile is shown in Fig. 2(b) for the $\mathrm{Si} / \mathrm{Ge} / \mathrm{Si}$ structure with $L_{F}=10.6 \mathrm{~nm}$. To minimize the uncertainty in specifying the temperature drop across the film, we apply a least-squares linear regression analysis to the temperature profile in each lead and evaluate the linear fits at the lead/ film boundaries. The nonlinear regions in the temperature profile found in the 100 monolayers closest to each reservoir/ lead boundary are neglected when performing the regression analysis, as shown in Fig. 2(b).

\section{RESULTS}

\section{A. Thin film thermal resistance}

The LD- and MD-predicted thin film thermal resistances are provided in Fig. 3. Error bars representing the 95\% confidence interval based on five independent simulations are 


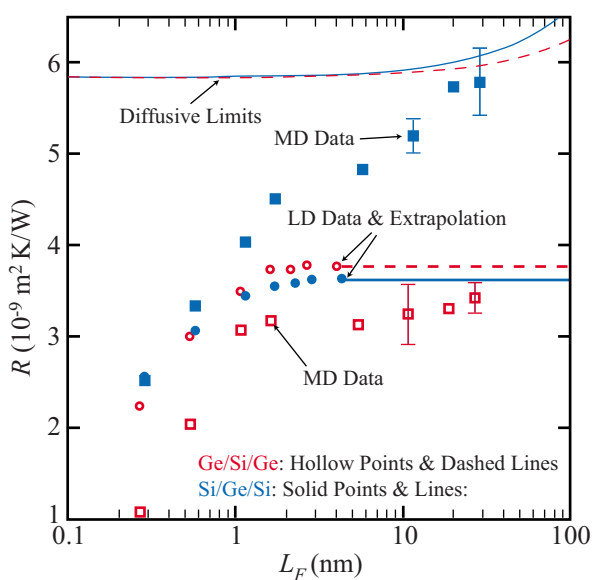

FIG. 3. (Color online) Thin film thermal resistances predicted from LDbased calculations and MD simulations. The LD-based calculations are performed in the classical limit for comparison to the classical MD simulations, which are performed at a temperature of $500 \mathrm{~K}$. The thin film thermal resistance in the diffusive limit is also provided for comparison.

provided for four of the MD-predicted data points. The MD predictions are made for films with thicknesses between 0.2 and $30 \mathrm{~nm}$, while the LD predictions are made for thicknesses between 0.2 and $4 \mathrm{~nm}$. Because the LD-predicted thin film thermal resistances are independent of the film thickness for $L_{F}>2 \mathrm{~nm}$, however, we extrapolate the values at $L_{F}$ $=4 \mathrm{~nm}$ to allow for comparison to the MD predictions. The values of the thin film thermal resistance in the diffusive limit, $R_{\mathrm{DL}}$, reached when the film thickness is much greater than the phonon mean free paths in the corresponding bulk material, are also provided in Fig. 3 for comparison. The thin film thermal resistance in the diffusive limit is

$$
R_{\mathrm{DL}}=2 R_{\mathrm{Si} / \mathrm{Ge}}+\frac{L_{F}}{k},
$$

where $R_{\mathrm{Si} / \mathrm{Ge}}$ is the $\mathrm{Si} / \mathrm{Ge}$ thermal boundary resistance and $k$ is the bulk thermal conductivity of the film species. The $L_{F} / k$ term is the thermal conduction resistance often used in continuum-level heat transfer analyses. ${ }^{47}$ For our calculation of the diffusive limit, $R_{\mathrm{Si} / \mathrm{Ge}}$ is predicted using direct method MD simulations and $k_{\mathrm{Si}}$ and $k_{\mathrm{Ge}}$ are predicted using $\mathrm{MD}$ simulations and the Green-Kubo method. The predicted values of $R_{\mathrm{Si} / \mathrm{Ge}}, k_{\mathrm{Si}}$, and $k_{\mathrm{Ge}}$ at a temperature of $500 \mathrm{~K}$ are $2.93 \pm 0.29 \times 10^{-9} \mathrm{~m}^{2} \mathrm{~K} / \mathrm{W}, \quad 230 \pm 47 \mathrm{~W} / \mathrm{m} \mathrm{K}, \quad$ and $132 \pm 34 \mathrm{~W} / \mathrm{m} \mathrm{K}^{18,44}$

For the $\mathrm{Ge} / \mathrm{Si} / \mathrm{Ge}$ structures, the MD-predicted thin film thermal resistances increase rapidly with increasing thickness for $L_{F}<2 \mathrm{~nm}$ and are independent of thickness for $2 \mathrm{~nm}$ $<L_{F}<30 \mathrm{~nm}$. This trend is in qualitative agreement with that predicted from LD. The LD-predicted thin film thermal resistances, however, are greater than the MD-predicted values. For example, the LD-predicted thermal resistance of the $\mathrm{Ge} / \mathrm{Si} / \mathrm{Ge}$ structure with $L_{F}=0.27 \mathrm{~nm}$ is two times greater than the MD-predicted value. We believe that this discrepancy is due to inaccuracy in the phonon distribution functions used in the LD calculations because the MD-predicted thin film thermal resistance is less than the $\mathrm{Si} / \mathrm{Ge}$ thermal boundary resistance [see discussion preceding Eq. (4)]. For the structures with $L_{F}>2 \mathrm{~nm}$, the MD-predicted thin film thermal resistances are greater than the $\mathrm{Si} / \mathrm{Ge}$ thermal boundary resistance, and the LD- and MD-predicted thermal resistances agree to within $15 \%$.

For the $\mathrm{Si} / \mathrm{Ge} / \mathrm{Si}$ structures with $L_{F}<2 \mathrm{~nm}$, the MDpredicted thin film thermal resistances increase rapidly with increasing thickness. This trend is in agreement with that predicted from the LD calculations. Differences are observed between the MD and LD predictions, however, for structures with $L_{F}>2 \mathrm{~nm}$. For these structures, the MD-predicted thin film thermal resistances continue to increase toward the diffusive limit with increasing film thickness, while the LDpredicted values are independent of the film thickness.

We propose two mechanisms for understanding the thickness dependence of the thin film thermal resistances. The first mechanism is changes in the allowed vibrational states in the film, which can influence both the phonon transmission coefficients and the nature of the phonon-phonon scattering in the film (through the selection rules for multiphonon scattering events). Such changes arise due to the decreasing fraction of film atoms that are bonded to lead atoms and the increased resolution of the Brillouin zone of the film species (defined using the bulk primitive lattice vectors) as the film thickness increases. The second mechanism is increased phonon-phonon scattering in the film as the film thickness approaches or exceeds the mean free path of the phonons in the film. Such inelastic scattering leads to reduced coupling of phonons on either side of the film, which may alter the phonon transmission coefficients, and increased thermal conduction resistance of the film. In Secs. III B and III C, we will discuss how these two mechanisms affect the thermal resistance of the $\mathrm{Si} / \mathrm{Ge} / \mathrm{Si}$ and $\mathrm{Ge} / \mathrm{Si} / \mathrm{Ge}$ structures.

\section{B. Allowed vibrational state effect}

Because the LD calculations neglect inelastic phonon scattering, the thickness dependence of the LD-predicted thin film thermal resistances must be due to changes in the allowed vibrational states in the film. The MD-predicted thickness dependence for the $\mathrm{Si} / \mathrm{Ge} / \mathrm{Si}$ structures with $L_{F}<2 \mathrm{~nm}$ and all of the $\mathrm{Ge} / \mathrm{Si} / \mathrm{Ge}$ structures is also likely to be a result of these changes due to the qualitative agreement between the LD-and MD-predicted trends. To quantify the thickness dependence of the allowed vibrational states, we use LD calculations to obtain the phonon density of states (PDOS), $\rho(\omega)$, in the film. These calculations are performed using a unit cell that contains one atom for each monolayer in the film and one atom for each of 12 monolayers in each lead. This unit cell is periodically replicated in the $x-y$ plane. Increasing the length of each lead has no discernible effect on the PDOS in the film. Extending on the concept of a local PDOS (i.e., the PDOS of a single atom in a supercell) used by others, ${ }^{48}$ we define the PDOS in the film to be

$$
\rho\left(\omega^{\prime}\right)=\frac{1}{(2 \pi)^{2} L_{F}} \int \sum_{\nu} \sum_{j} \sum_{\beta} \delta\left[\omega(\boldsymbol{\kappa}, \nu)-\omega^{\prime}\right]\left|e_{j, \beta}(\boldsymbol{\kappa}, \nu)^{2}\right| d \boldsymbol{\kappa},
$$

where the $j$-summation is over the film atoms in the unit cell, the $\beta$-summation is over the three Cartesian coordinates, 


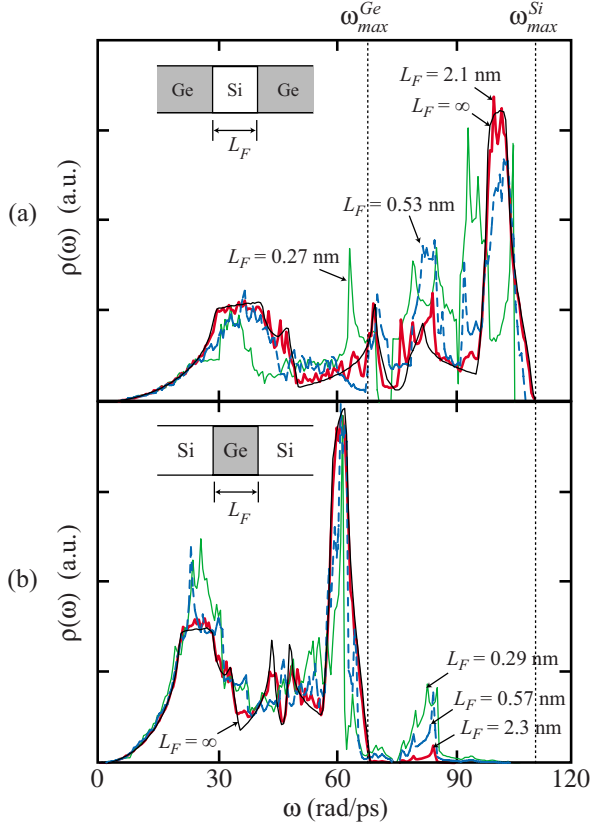

FIG. 4. (Color online) PDOS in the films of (a) three $\mathrm{Ge} / \mathrm{Si} / \mathrm{Ge}$ and (b) three $\mathrm{Si} / \mathrm{Ge} / \mathrm{Si}$ structures. The PDOS for bulk $\mathrm{Si}$ and $\mathrm{Ge}$ (denoted by $L_{F}=\infty$ ) are also provided for comparison.

$e_{j, \beta}(\boldsymbol{\kappa}, \nu)$ is the $j, \beta$-component of the phonon polarization vector for mode $(\boldsymbol{\kappa}, \nu)$, and

$$
\begin{aligned}
\delta & =\frac{1}{\Delta \omega} \quad \text { if }\left|\omega(\boldsymbol{\kappa}, \nu)-\omega^{\prime}\right| \leq \frac{\Delta \omega}{2} \\
& =0 \quad \text { otherwise. }
\end{aligned}
$$

When the PDOS is defined using Eq. (6), its integral over frequency is equal to $3 n$, where $n$ is the number density of atoms in the film. We evaluate Eq. (6) using Monte Carlo integration with $10^{4}$ random phonon wavevectors in the twodimensional Brillouin zone for the thin film structure and a frequency bin width, $\Delta \omega$, of $0.5 \mathrm{rad} / \mathrm{ps}$.

The PDOS in the films of three Ge/Si/Ge and three Si/ $\mathrm{Ge} / \mathrm{Si}$ structures are provided in Figs. 4(a) and 4(b). The PDOS for bulk $\mathrm{Si}$ and $\mathrm{Ge}$, which are obtained under the same strain conditions that exist in the thin film structures, are also provided for comparison (denoted by $L_{F}=\infty$ ). For films with $L_{F}<2 \mathrm{~nm}$, the film PDOS is different than that of the corresponding bulk PDOS. Notable is the nonzero PDOS in the $\mathrm{Ge}$ film of the $\mathrm{Si} / \mathrm{Ge} / \mathrm{Si}$ structure between the maximum frequency in bulk Ge, $\omega_{\max }^{\mathrm{Ge}}$, of $69 \mathrm{rad} / \mathrm{ps}$ and a frequency of $\sim 90 \mathrm{rad} / \mathrm{ps}$ [Fig. 4(b)]. As the film thickness increases, the PDOS in the film becomes increasingly bulklike, with minimal differences between the film and bulk PDOS observed for films with thickness $L_{F}>2 \mathrm{~nm}$. This transition to a bulklike PDOS coincides with the thickness where the thin film thermal resistances stop increasing rapidly with increasing film thickness. We take this agreement as evidence that the thickness dependence of the thin film thermal resistance for films with $L_{F}<2 \mathrm{~nm}$ is due to changes in the allowed vibrational states in the film.

We further examine how the changes in the allowed vibrational states in the film affect the thermal transport by calculating the contributions to the LD-predicted thin film thermal conductance, $G_{\mathrm{LD}}$, as a function of either phonon frequency or incidence angle, $\theta$. These functions, $g_{\omega}$ and $g_{\theta}$, are defined such that

$$
G_{\mathrm{LD}}=R_{\mathrm{LD}}^{-1}=\int_{0}^{\infty} g_{\omega} d \omega=\int_{0}^{\pi / 2} g_{\theta} d \theta
$$

In Figs. 5(a)-5(d), $g_{\omega}$ and $g_{\theta}$ are provided for the thin film
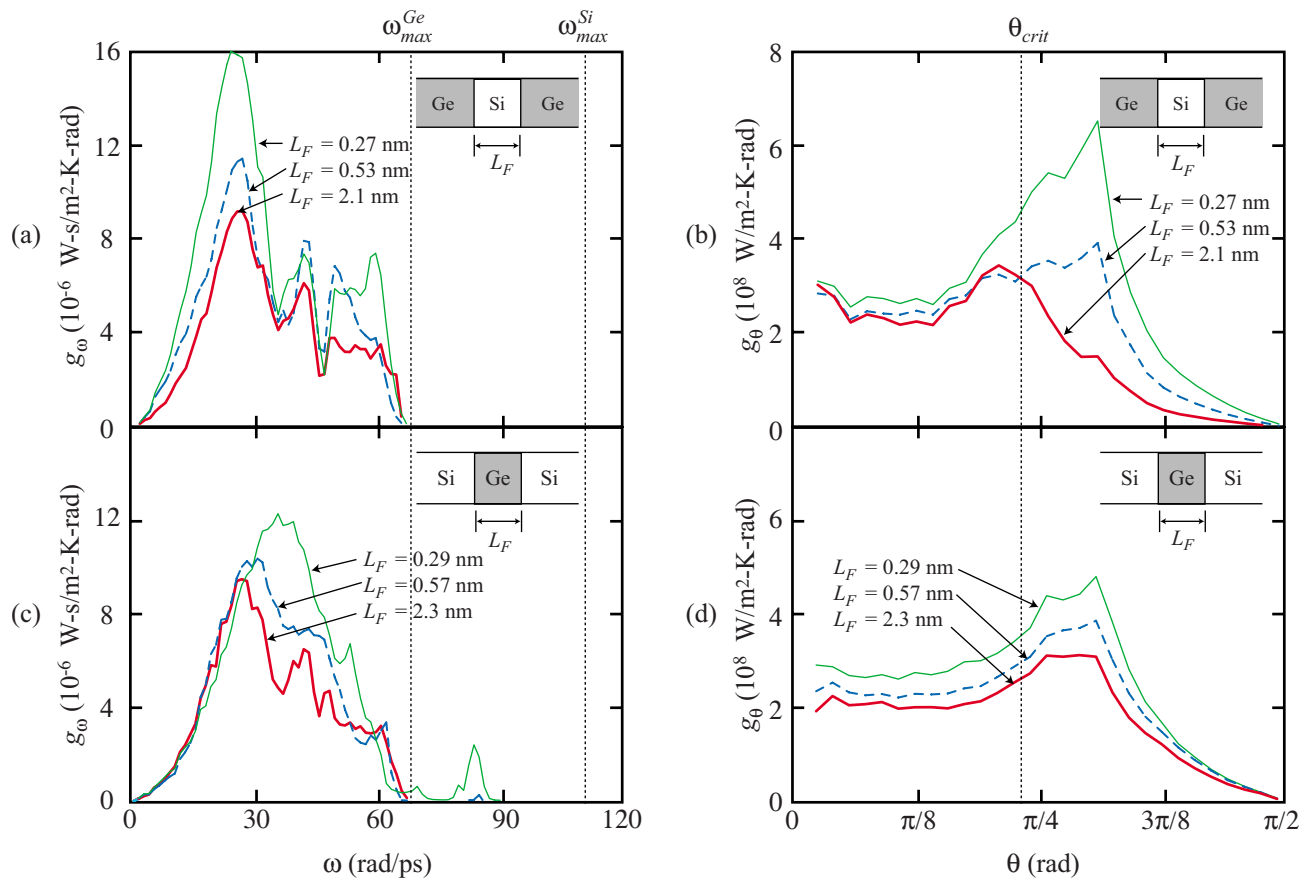

FIG. 5. (Color online) Dependence of the LD-predicted thin film thermal conductance on (a) phonon frequency and (b) phonon incidence angle for the $\mathrm{Ge} / \mathrm{Si} / \mathrm{Ge}$ structures and (c) phonon frequency and (d) phonon incidence angle for the $\mathrm{Si} / \mathrm{Ge} / \mathrm{Si}$ structures. For thin film structures with $L_{F}>2 \mathrm{~nm}, g_{\omega}$ and $g_{\theta}$ are independent of film thickness. 
structures for which we provided the PDOS in Figs. 4(a) and 4(b). As with the film PDOS, $g_{\omega}$ and $g_{\theta}$ are independent of film thickness for $L_{F}>2 \mathrm{~nm}$. Vertical lines are provided in Figs. 5(a) and 5(c) for the maximum phonon frequencies in bulk $\mathrm{Si}$ and $\mathrm{Ge}$. We also provide a vertical line for the $\mathrm{Si} / \mathrm{Ge}$ critical angle, $\theta_{\text {crit }}$, in Figs. 5(b) and 5(d). By Snell's law, the phonon transmission coefficient is zero for a phonon incident on an interface from a material with a low group velocity to a material with higher group velocity (e.g., Ge to $\mathrm{Si}$ ) if its incidence angle is greater than $\theta_{\text {crit }} \cdot{ }^{10,36,42}$ We estimate $\theta_{\text {crit }}$ to be $0.73 \mathrm{rad}$ under the approximation that the phonon group velocities in each material are equal to the speed of sound, $c$, which we take to be the average of the three [001] acoustic phonon group velocities in the $\boldsymbol{\kappa} \rightarrow 0$ limit. Due to the realistic phonon dispersion used in our LD calculations, $g_{\theta}$ is nonzero for incidence angles greater than this estimated $\theta_{\text {crit }}$. We thus use $\theta_{\text {crit }}$ only as a point of reference.

While the LD-predicted thermal resistances of the Ge/ $\mathrm{Si} / \mathrm{Ge}$ and $\mathrm{Si} / \mathrm{Ge} / \mathrm{Si}$ structures are comparable in terms of magnitude and thickness dependence, the thickness dependence is manifested differently in each type of structure. As the film thickness in the $\mathrm{Ge} / \mathrm{Si} / \mathrm{Ge}$ structures decreases, $g_{\theta}$ significantly increases only for $\theta$ greater than $\sim \theta_{\text {crit }}$ [see Fig. $5(\mathrm{~b})]$, and $g_{\omega}$ increases for all frequencies by an approximately frequency-independent factor [Fig. 5(a)]. As the film thickness in the $\mathrm{Si} / \mathrm{Ge} / \mathrm{Si}$ structures decreases, $g_{\omega}$ increases only for $\omega$ greater than $\sim 30 \mathrm{rad} / \mathrm{ps}$ [Fig. 5(c)], and $g_{\theta}$ increases for all incident angles by a factor that is independent of incidence angle [Fig. 5(d)]. For these structures, $g_{\omega}$ is even nonzero for frequencies of $\omega_{\max }^{\mathrm{Ge}}<\omega<90 \mathrm{rad} / \mathrm{ps}$ due to the vibrational states that exist in this frequency range when $L_{F}<2 \mathrm{~nm}$ [see Fig. 4(b)]. These trends are due solely to changes in the phonon transmission coefficients because the other phonon properties relevant to the thermal conductance [see Eq. (4)] are independent of the film thickness. Furthermore, the frequency and angular dependences of the phonon transmission coefficients can be separated because there is little correlation between phonon direction and frequency in bulk Si and bulk $\mathrm{Ge}$, as evidenced by their near-isotropic phonon dispersion curves. ${ }^{49}$ The $g_{\omega}$ and $g_{\theta}$ trends therefore suggest that the changes in the allowed vibrational states affect mostly the transmission coefficients of (i) phonons with $\theta>\theta_{\text {crit }}$ in the $\mathrm{Ge} / \mathrm{Si} / \mathrm{Ge}$ structures (independent of frequency) and (ii) phonons with $\omega>30 \mathrm{rad} / \mathrm{ps}$ in the $\mathrm{Si} / \mathrm{Ge} / \mathrm{Si}$ structures (independent of incidence angle).

\section{Phonon-phonon scattering effect}

\section{Temperature profiles}

As demonstrated in Sec. III B, thickness-dependent changes in the allowed vibrational states have negligible effect on the thin film thermal resistance for films with $L_{F}$ $>2 \mathrm{~nm}$. Any thickness dependence of the thin film thermal resistance for these films must therefore be due to the effects of inelastic phonon-phonon scattering in the film. Because the MD-predicted thermal resistances of the $\mathrm{Ge} / \mathrm{Si} / \mathrm{Ge}$ structures with $2<L_{F}<30 \mathrm{~nm}$ are thickness independent, we believe that the phonon transport is ballistic in the Si film. For the $\mathrm{Si} / \mathrm{Ge} / \mathrm{Si}$ structures with $L_{F}>2 \mathrm{~nm}$, however, the MD-

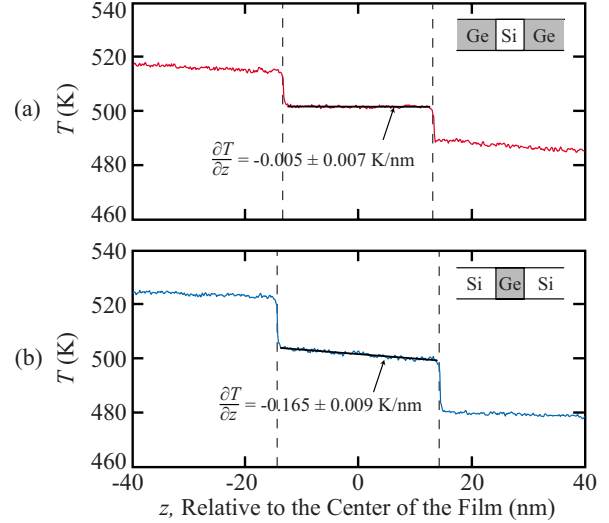

FIG. 6. (Color online) Steady-state temperature profiles near and across the thin film region of the $\mathrm{Si} / \mathrm{Ge} / \mathrm{Si}$ and $\mathrm{Ge} / \mathrm{Si} / \mathrm{Ge}$ structures with a film thickness of 200 monolayers $\left(L_{F}=28.7 \mathrm{~nm}\right.$ for the Ge film and $L_{F}=26.6 \mathrm{~nm}$ for the $\mathrm{Si}$ film) predicted from MD simulation. The same heat flux of $q$ $=7.23 \mathrm{GW} / \mathrm{m}^{2}$ was applied across both structures.

predicted thin film thermal resistances increase with increasing film thickness, suggesting that the phonon-phonon scattering in the Ge film is non-negligible (i.e., the phonon transport is partially diffusive). Because the LD calculations assume ballistic phonon transport, this argument also explains why the LD- and MD-predicted thin film thermal resistances are in good agreement for the $\mathrm{Ge} / \mathrm{Si} / \mathrm{Ge}$ structures but are in poor agreement for the $\mathrm{Si} / \mathrm{Ge} / \mathrm{Si}$ structures with $L_{F}>2 \mathrm{~nm}$.

Additional evidence for ballistic phonon transport in the $\mathrm{Ge} / \mathrm{Si} / \mathrm{Ge}$ structures and more diffusive transport in the $\mathrm{Si} /$ $\mathrm{Ge} / \mathrm{Si}$ structures is found in the MD-predicted steady-state temperature profiles. The temperature profiles across a $\mathrm{Ge} /$ $\mathrm{Si} / \mathrm{Ge}$ structure with $L_{F}=26.6 \mathrm{~nm}$ and a $\mathrm{Si} / \mathrm{Ge} / \mathrm{Si}$ structure with $L_{F}=28.7 \mathrm{~nm}$ (the films in both structures have a thickness of 200 monolayers) are shown in Figs. 6(a) and 6(b) as an example. The same heat flux of $q=7.23 \mathrm{GW} / \mathrm{m}^{2}$ was applied across both structures. To reduce statistical fluctuations, each temperature profile is averaged over five independent simulations. Using a least-squares linear regression analysis, we find that the temperature gradient in the Si film is $-0.005 \pm 0.007 \mathrm{~K} / \mathrm{nm}$ (95\% confidence). This value is a factor of 6 less than the value of $-0.031 \mathrm{~K} / \mathrm{nm}$ that would develop in $\mathrm{Si}$ if the phonon scattering was entirely diffusive [calculated from the Fourier law, $q=-k(\partial T / \partial z)]$. Because phonon scattering is required to establish a temperature gradient, ${ }^{11,49-51}$ we take the small temperature gradient in the Si film relative to the value in the diffusive limit as evidence of ballistic phonon transport. In the Ge film, the temperature gradient is $-0.165 \pm 0.009 \mathrm{~K} / \mathrm{nm}$ ( $95 \%$ confidence). This temperature gradient is a factor of 3 greater than the value of $-0.060 \mathrm{~K} / \mathrm{nm}$ that would develop if the phonon scattering was entirely diffusive, indicating the presence of phononphonon scattering in the film.

The MD-predicted thickness dependence of the film temperature gradient for a fixed value of the heat flux is also different between the $\mathrm{Ge} / \mathrm{Si} / \mathrm{Ge}$ and $\mathrm{Si} / \mathrm{Ge} / \mathrm{Si}$ structures. For all of the $\mathrm{Ge} / \mathrm{Si} / \mathrm{Ge}$ structures $\left(L_{F}<30 \mathrm{~nm}\right)$, the temperature gradient is nearly zero and has no thickness dependence because the phonon transport is ballistic [see Fig. 6(a)]. Flat 
temperature profiles have also been predicted by others using the lattice Boltzmann method for films in which the phonon transport is ballistic. ${ }^{49,50}$ As the film thickness increases, the lattice Boltzmann method predicts that the magnitude of the film temperature gradient increases toward the value in the diffusive limit as the phonon transport becomes increasingly diffusive. We expect that a similar trend would be observed for $\mathrm{Ge} / \mathrm{Si} / \mathrm{Ge}$ structures with $L_{F}>30 \mathrm{~nm}$. For the $\mathrm{Si} / \mathrm{Ge} / \mathrm{Si}$ structures, however, the opposite trend is observed, and the magnitude of the temperature gradient in the Ge film decreases toward the value in the diffusive limit as the film thickness increases. This trend is in agreement with that predicted by others using MD simulation for films confined by two thermal reservoirs of the same species. ${ }^{19-23}$ This difference suggests that the thermal transport in thin films depends not only on the species and thickness of the film but also on the materials that confine it.

\section{Transition between ballistic and diffusive phonon transport}

We now seek to determine why ballistic phonon transport is observed in the Ge/Si/Ge structures while more diffusive transport is observed in the $\mathrm{Si} / \mathrm{Ge} / \mathrm{Si}$ structures. The standard technique for determining whether the phonon transport is ballistic, diffusive, or partially diffusive is to compare the average bulk phonon mean free path, $\Lambda$, to the film thickness. An estimate for $\Lambda$ is obtained from the kinetic theory expression for thermal conductivity, ${ }^{52}$

$$
k=\frac{1}{3} C_{v} c \Lambda,
$$

where $C_{v}$ is the specific heat per unit volume. Using thermal conductivities predicted from Green-Kubo MD simulations, classical specific heats, and our approximate sound speeds (see Sec. III B), we estimate $\Lambda$ for both bulk Si and Ge to be $50 \mathrm{~nm}$ at a temperature of $500 \mathrm{~K}$. Based on this result, the transition from ballistic to diffusive transport might be expected to occur for similar values of the film thickness in the $\mathrm{Ge} / \mathrm{Si} / \mathrm{Ge}$ and $\mathrm{Si} / \mathrm{Ge} / \mathrm{Si}$ structures, which is contrary to our MD predictions (see Fig. 3). A higher-level analysis of the phonon-phonon scattering in the bulk species is needed.

We examine the details of the phonon-phonon scattering in bulk Si and Ge by calculating the phonon mean free path as a function of phonon frequency $\bar{\lambda}_{z}(\omega)$ at a temperature of $500 \mathrm{~K}$. We define $\bar{\lambda}_{z}(\omega)$ to be

$$
\bar{\lambda}_{z}\left(\omega^{\prime}\right)=\frac{\int \sum_{\nu} \lambda_{z}(\boldsymbol{\kappa}, \nu) \delta\left[\omega(\boldsymbol{\kappa}, \nu)-\omega^{\prime}\right] d \boldsymbol{\kappa}}{\int \sum_{\nu} \delta\left[\omega(\boldsymbol{\kappa}, \nu)-\omega^{\prime}\right] d \boldsymbol{\kappa}} .
$$

Here, $\lambda_{z}$ is the mode-dependent phonon mean free path in the $z$-direction, which is equal to $v_{z} \tau$, where $\tau$ is the modedependent phonon relaxation time. We evaluate Eq. (10) using a frequency bin width of $1 \mathrm{rad} / \mathrm{ps}$ and phonon relaxation times predicted using anharmonic LD calculations for $32^{3}$ evenly spaced wavevectors in the first Brillouin zone for the conventional diamond unit cell. ${ }^{15,53}$ The results are plotted in Fig. 7.

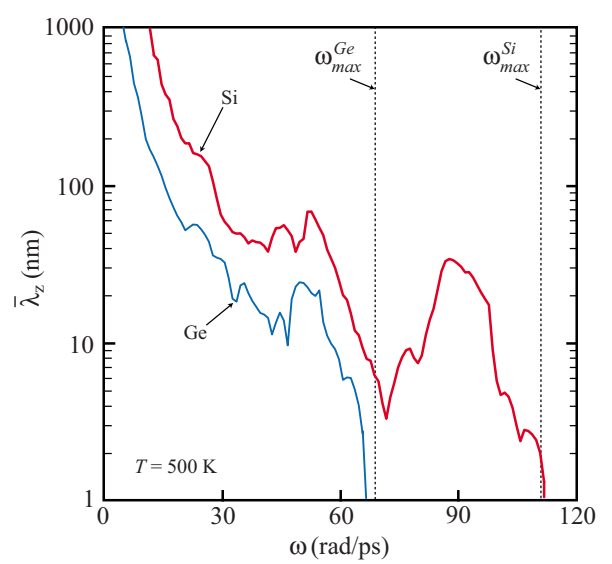

FIG. 7. (Color online) Average phonon mean free path in bulk Si and Ge as a function of phonon frequency at a temperature of $500 \mathrm{~K}$.

Consider $\mathrm{Si} / \mathrm{Ge} / \mathrm{Si}$ and $\mathrm{Ge} / \mathrm{Si} / \mathrm{Ge}$ structures with film thicknesses of $L_{F}=1 \mathrm{~nm}$. As shown in Fig. $7, \bar{\lambda}_{z}$ is greater than this film thickness for all phonon frequencies in bulk $\mathrm{Ge}$ and bulk Si. The phonon transport in both films is thus ballistic. Now consider what happens as the film thickness increases. For the $\mathrm{Si} / \mathrm{Ge} / \mathrm{Si}$ structure, the film thickness will immediately begin to exceed $\bar{\lambda}_{z}$ in bulk Ge for frequencies near $\omega_{\max }^{\mathrm{Ge}}$, leading to partially diffusive transport. When the film thickness reaches $L_{F}=28.7 \mathrm{~nm}$ (our largest value for the $\mathrm{Si} / \mathrm{Ge} / \mathrm{Si}$ structure), the film thickness exceeds $\bar{\lambda}_{z}$ for $\omega$ $>31 \mathrm{rad} / \mathrm{ps}$. According to Fig. 5(c), phonons in this frequency range contribute $51 \%$ to the thermal conductance when the phonon transport is ballistic [the LD calculations used to generate Figs. 5(a)-5(d) assume ballistic transport]. For the $\mathrm{Ge} / \mathrm{Si} / \mathrm{Ge}$ structure, the phonon transport is entirely ballistic until the film thickness exceeds $\bar{\lambda}_{z}\left(\omega_{\max }^{\mathrm{Ge}}\right)=5 \mathrm{~nm}$ because phonons with $\omega>\omega_{\max }^{\mathrm{Ge}}$ do not contribute to the thermal transport [see Fig. 5(a)]. When the film thickness reaches $L_{F}=26.6 \mathrm{~nm}$ (our largest value for the $\mathrm{Ge} / \mathrm{Si} / \mathrm{Ge}$ structure), the film thickness exceeds $\bar{\lambda}_{z}$ for $\omega>59 \mathrm{rad} / \mathrm{ps}$. According to Fig. 5(a), phonons in this frequency range contribute only $7 \%$ to the thermal conductance when the phonon transport is ballistic. These results suggest that the phonon transport across the $\mathrm{Si} / \mathrm{Ge} / \mathrm{Si}$ structures should be more diffusive than that across the $\mathrm{Ge} / \mathrm{Si} / \mathrm{Ge}$ structures, which is consistent with our MD predictions of the thin film thermal resistance (see Sec. III A). We note that for both types of thin film structure, fully diffusive phonon transport is not expected until the film thickness is $\sim 10 \mu \mathrm{m}$ due to the large phonon mean free paths at low frequencies and the lack of other scattering mechanisms in our models. ${ }^{49}$

\section{SUMMARY AND CONCLUSIONS}

We used LD calculations and MD simulations to predict the thin film thermal resistances of $\mathrm{Ge} / \mathrm{Si} / \mathrm{Ge}$ and $\mathrm{Si} / \mathrm{Ge} / \mathrm{Si}$ structures with film thicknesses, $L_{F}$, between 0.2 and $30 \mathrm{~nm}$. The LD calculations were performed in the classical limit for comparison to the classical MD simulations, which were performed at a temperature of $500 \mathrm{~K}$.

For structures with $L_{F}<2 \mathrm{~nm}$, the thin film thermal resistances increase rapidly with increasing film thickness (see 
Fig. 3). Because the PDOS in the film is thickness dependent only in this range (Fig. 4), we attributed this trend to changes in the allowed vibrational states in the film. We found that these changes affect the dependence of the phonon transmission coefficient on incidence angle for the $\mathrm{Ge} / \mathrm{Si} / \mathrm{Ge}$ structures and on frequency for the $\mathrm{Si} / \mathrm{Ge} / \mathrm{Si}$ structures [Figs. $5(\mathrm{a})-5(\mathrm{~d})]$.

For $\mathrm{Ge} / \mathrm{Si} / \mathrm{Ge}$ structures with $L_{F}>2 \mathrm{~nm}$, the MDpredicted thermal resistances are thickness independent and agree with the LD-predicted values to within $15 \%$. For $\mathrm{Si} /$ $\mathrm{Ge} / \mathrm{Si}$ structures with $L_{F}>2 \mathrm{~nm}$, the MD-predicted thermal resistances increase with increasing film thickness, while the LD-predicted values are thickness independent. Because the LD calculations assume that the phonon transport is ballistic, these results indicate that the phonon transport is ballistic in the $\mathrm{Ge} / \mathrm{Si} / \mathrm{Ge}$ structures and more diffusive in the $\mathrm{Si} / \mathrm{Ge} / \mathrm{Si}$ structures. We found that the standard technique of comparing the mode-averaged phonon mean free path to the film thickness is insufficient in predicting whether the phonon transport would be ballistic or diffusive in the film. The correct prediction can be made when the frequency dependence of the phonon mean free paths is considered (see Fig. 7).

The manner by which the temperature gradient in the film converges to the value in the diffusive limit for a fixed value of the heat flux was also found to be different between the two types of structure. For $\mathrm{Si} / \mathrm{Ge} / \mathrm{Si}$ structures, the magnitude of the temperature gradient decreases toward the value in the diffusive limit as the film thickness increases, while the opposite trend is expected for $\mathrm{Ge} / \mathrm{Si} / \mathrm{Ge}$ structures. We take this difference, along with the difference in the thickness dependence of the phonon transmission coefficients, as evidence that the thermal transport properties of a thin film depend on the materials that confines it in addition to the species and thickness of the film. This finding has implications in the interpretation of experimental data because it indicates that measured thermal conductivities may depend on the substrate material.

\section{ACKNOWLEDGMENTS}

We thank J. E. Turney (CMU) for providing the phonon relaxation times.

${ }^{1}$ D. Roberts and G. Triplett, Solid-State Electron. 52, 1669 (2008).

${ }^{2}$ Y. L. Li, Y. R. Huang, and Y. H. Lai, Appl. Phys. Lett. 91, 181113 (2007).

${ }^{3}$ S. Lombardo, J. H. Stathis, B. P. Linder, K. L. Pey, F. Palumbo, and C. H. Tung, J. Appl. Phys. 98, 121301 (2005).

${ }^{4}$ G. Chen, M. S. Dresselhaus, G. Dresselhaus, J.-P. Fleurial, and T. Caillat, Int. Mater. Rev. 48, 45 (2003).

${ }^{5} \mathrm{H}$. Bottner, G. Chen, and R. Venkatasubramanian, MRS Bull. 31, 211 (2006).

${ }^{6}$ M. S. Dresselhaus, G. Chen, M. Y. Tang, R. G. Yang, H. Lee, D. Z. Wang, Z. F. Ren, J. Fleurial, and P. Gogna, Adv. Mater. (Weinheim, Ger.) 19, 1043 (2007).

${ }^{7}$ Y. S. Ju and K. E. Goodson, Appl. Phys. Lett. 74, 3005 (1999).

${ }^{8}$ D. G. Cahill, W. K. Ford, K. E. Goodson, G. D. Mahan, A. Mujumdar, H. J. Maris, R. Merlin, and S. R. Phillpot, J. Appl. Phys. 93, 793 (2003).

${ }^{9}$ G. Chen and T. Zeng, Microscale Thermophys. Eng. 5, 71 (2001).

${ }^{10}$ G. Chen, Phys. Rev. B 57, 14958 (1998).
${ }^{11}$ C. V. D. R. Anderson and K. K. Tamma, Int. J. Numer. Methods Heat Fluid Flow 14, 12 (2004).

${ }^{12}$ A. D. McConnell and K. E. Goodson, Annu. Rev. Heat Transfer 14, 129 (2005).

${ }^{13}$ J. M. Ziman, Electrons and Phonons (Oxford University Press, New York, 2001).

${ }^{14}$ G. P. Srivastava, The Physics of Phonons (Hilger, Bristol, 1990).

${ }^{15}$ J. E. Turney, A. J. H. McGaughey, and C. H. Amon, Phys. Rev. B 79, 224305 (2009).

${ }^{16}$ Y. Chen, D. Li, J. R. Lukes, Z. Ni, and M. Chen, Phys. Rev. B 72, 174302 (2005).

${ }^{17}$ H. Zhao and J. B. Freund, J. Appl. Phys. 97, 024903 (2005).

${ }^{18}$ E. S. Landry and A. J. H. McGaughey, Phys. Rev. B 80, 165304 (2009).

${ }^{19}$ J. R. Lukes, D. Y. Li, X.-G. Liang, and C.-L. Tien, J. Heat Transfer 122, 536 (2000)

${ }^{20}$ P. K. Schelling, S. R. Phillpot, and P. Keblinski, Phys. Rev. B 65, 144306 (2002).

${ }^{21}$ P. Chantrenne and J. L. Barrat, J. Heat Transfer 126, 577 (2004).

${ }^{22}$ X. L. Feng, Nanoscale Microscale Thermophys. Eng. 7, 153 (2003).

${ }^{23}$ Z. Huang, Z. Tang, J. Yu, and S. Bai, Physica B 404, 1790 (2009).

${ }^{24}$ P. K. Schelling, S. R. Phillpot, and P. Keblinski, Appl. Phys. Lett. 80, 2484 (2002).

${ }^{25}$ A. J. H. McGaughey and M. Kaviany, Phys. Rev. B 69, 094303 (2004).

${ }^{26}$ J. Shiomi and S. Maruyama, Int. J. Thermophys. (2008).

${ }^{27}$ M. T. Dove, Introduction to Lattice Dynamics (Cambridge University Press, Cambridge, 1993).

${ }^{28}$ W. Zhang, T. S. Fisher, and N. Mingo, J. Heat Transfer 129, 483 (2007).

${ }^{29}$ While we chose lattice constants to minimize the strain associated with the lattice mismatch between $\mathrm{Si}$ and $\mathrm{Ge}$, we find that the effect of strain on the thermal resistance of Si- and Ge-based interfaces and films is minor. For example, the thermal boundary resistance of the symmetrically strained $\mathrm{Si} / \mathrm{Ge}$ interface was calculated in our earlier work to be 3.0 $\times 10^{-9} \mathrm{~m}^{2} \mathrm{~K} / \mathrm{W}$ in the classical limit (Ref. 18). This value is within $5 \%$ of the value calculated by Zhao and Freund (Ref. 17), who neglected the strain associated with the lattice mismatch.

${ }^{30}$ F. H. Stillinger and T. A. Weber, Phys. Rev. B 31, 5262 (1985).

${ }^{31}$ K. Ding and H. C. Andersen, Phys. Rev. B 34, 6987 (1986).

${ }^{32}$ M. Laradji, D. P. Landau, and B. Dunweg, Phys. Rev. B 51, 4894 (1995).

${ }^{33}$ C. Kimmer, S. Aubry, A. Skye, and P. K. Schelling, Phys. Rev. B 75, 144105 (2007).

${ }^{34}$ S. Aubry, C. J. Kimmer, A. Skye, and P. K. Schelling, Phys. Rev. B 78, 064112 (2008).

${ }^{35}$ D. A. Young and H. J. Maris, Phys. Rev. B 40, 3685 (1989).

${ }^{36}$ J. Wang and J. Wang, J. Phys.: Condens. Matter 19, 236211 (2007).

${ }^{37}$ S. Pettersson and G. D. Mahan, Phys. Rev. B 42, 7386 (1990).

${ }^{38}$ P. Reddy, K. Castelino, and A. Majumdar, Appl. Phys. Lett. 87, 211908 (2005).

${ }^{39}$ S. Simons, J. Phys. C 7, 4048 (1974).

${ }^{40}$ J. Wang, J. Wang, and J. T. Lu, Eur. Phys. J. B 62, 381 (2008).

${ }^{41}$ J. Wang and J. Wang, Phys. Rev. B 74, 054303 (2006).

${ }^{42}$ E. T. Swartz and R. O. Pohl, Rev. Mod. Phys. 61, 605 (1989).

${ }^{43}$ P. K. Schelling and S. R. Phillpot, J. Appl. Phys. 93, 5377 (2003).

${ }^{44}$ E. S. Landry, Ph.D. thesis, Carnegie Mellon University, 2009.

${ }^{45}$ A. J. H. McGaughey, M. I. Hussein, E. S. Landry, M. Kaviany, and G. M. Hulbert, Phys. Rev. B 74, 104304 (2006).

${ }^{46}$ T. Ikeshoji and B. Hafskjold, Mol. Phys. 81, 251 (1994).

${ }^{47}$ F. P. Incropera and D. P. DeWitt, Fundamentals of Heat and Mass Transfer (Wiley, Hoboken, NJ, 2002).

${ }^{48}$ Z. Tang and N. R. Aluru, Phys. Rev. B 74, 235441 (2006).

${ }^{49}$ D. P. Sellan, J. E. Turney, A. J. H. McGaughey, and C. H. Amon (unpublished).

${ }^{50}$ R. A. Escobar, S. S. Ghai, M. S. Jhon, and C. H. Amon, Int. J. Heat Mass Transfer 49, 97 (2006).

${ }^{51}$ G. Chen, J. Nanopart. Res. 2, 199 (2000).

${ }^{52}$ N. W. Ashcroft and N. D. Mermin, Solid State Physics (Saunders College, Fort Worth, TX, 1976).

${ }^{53}$ J. E. Turney, E. S. Landry, A. J. H. McGaughey, and C. H. Amon, Phys. Rev. B 79, 064301 (2009). 